\title{
Not tarsal tunnel syndrome: a malignant "Triton" tumour of the tibial nerve
}

\author{
C M WILES, S WHITEHEAD, A B WARD, C D M FLETCHER
}

From St Thomas' Hospital, London, UK

SUMMARY A 23 year old male patient presented with a venous thrombosis in the right calf. This was followed by symptoms, signs and electromyographic findings suggestive of a tarsal tunnel syndrome. Symptoms were briefly relieved by surgical division of the flexor retinaculum. Subsequently, patient developed swelling in the calf and was found to have a malignant ("Triton") tumour of the tibial nerve and required above knee amputation. In the absence of obvious foot or ankle deformity, caution should be exercised in making the diagnosis of tarsal tunnel syndrome.

Although tarsal tunnel syndrome is apparently a widely recognised entrapment neuropathy ${ }^{1}$ the case below illustrates that some caution should be exercised in making this diagnosis too readily.

A Caucasian male aged 23 years presented in 1983 with right calf pain, and swelling of the calf and foot. A venogram showed a deep vein thrombosis and following anticoagulant treatment the symptoms and signs resolved. A year later he presented with burning pains in the foot, alleviated by dependency and worsened by elevation, with numbness and paraesthesias in the sole. Examination showed slight oedema behind the medial malleolus, wasting of the small muscles of the foot (excluding extensor digitorum brevis), preserved reflexes, and sensory loss to light touch and pinprick in the distribution of the medial and lateral plantar nerves sparing the calcaneal and sural nerve distributions: Tinel's sign was positive over the tarsal tunnel and ischaemia of the lower leg exacerbated the symptoms. EMG studies showed denervation in the small foot muscles but normal calf muscles. Distal motor latency (ankle to abductor hallucis brevis) was $5.8 \mathrm{~ms}$ on the right and $3.5 \mathrm{~ms}$ on the left with a dispersed and small compound muscle action potential on the right: the right lateral plantar sensory action potential (SAP) was absent, the medial plantar SAP was $0.5 \mu \mathrm{v}$ (Right) and $1.8 \mu \mathrm{v}$ (Left), the sural SAP $9 \mu \mathrm{v}$. Venogram, arteriogram and CT scan of the right lower leg were normal. A right tarsal tunnel exploration was performed ( $\mathrm{Mr} \mathrm{A}$. E. Thompson) and

Address for reprint requests: $\mathrm{Dr}$ CM Wiles, Department of Neurology, St Thomas' Hospital, London SE1 7EH, UK.

Received 13 May 1986. Accepted 19 June 1986 revealed a pale swollen tibial nerve under a thick fibrous band which was divided. Post operatively the patient's symptoms resolved but this improvement was short-lived.

A month later the patient had recurrent pains in the foot which gradually increased over 3 months despite various medications. In June 1985 he developed obvious swelling of the whole lower leg with a haematoma above the medial malleolus. The haematoma was evacuated and the wall biopsied; the biopsy showed features of a malignant nerve sheath tumour. An above knee amputation was performed. The specimen showed, externally, an ulcerated mass measuring up to $12 \mathrm{~cm}$ in diameter situated $9 \mathrm{~cm}$ above the medical malleolus posteriorly. Dissection revealed that the tumour was arising from and destroying the tibial nerve $15 \mathrm{~cm}$ below the knee joint (fig a). The lesion, which was firm and pale, showed multifocal necrosis and haemorrhage and had extensively invaded subcutaneous tissues and skeletal muscle locally.

Histologically the main tumour mass showed typical features of a malignant nerve sheath tumour. It was mainly composed of interlacing fascicles of close packed spindle cells set in a loose rather myxoid stroma (fig b) with marked necrosis and haemorrhage: the tumour cells had elongated nuclei and indistinct cytoplasm: there was focal nuclear palisading and prominent perivascular whorling. Abnormal mitotic activity was prominent. In four out of 19 blocks of tumour tissue examined there were scattered variably small, elongated or large multinucleate cells with prominent eosinophilic cytoplasm: phosphotungstic acid haematoxylin stain revealed numerous intracytoplasmic cross-striations (fig c) indicating the pres- 

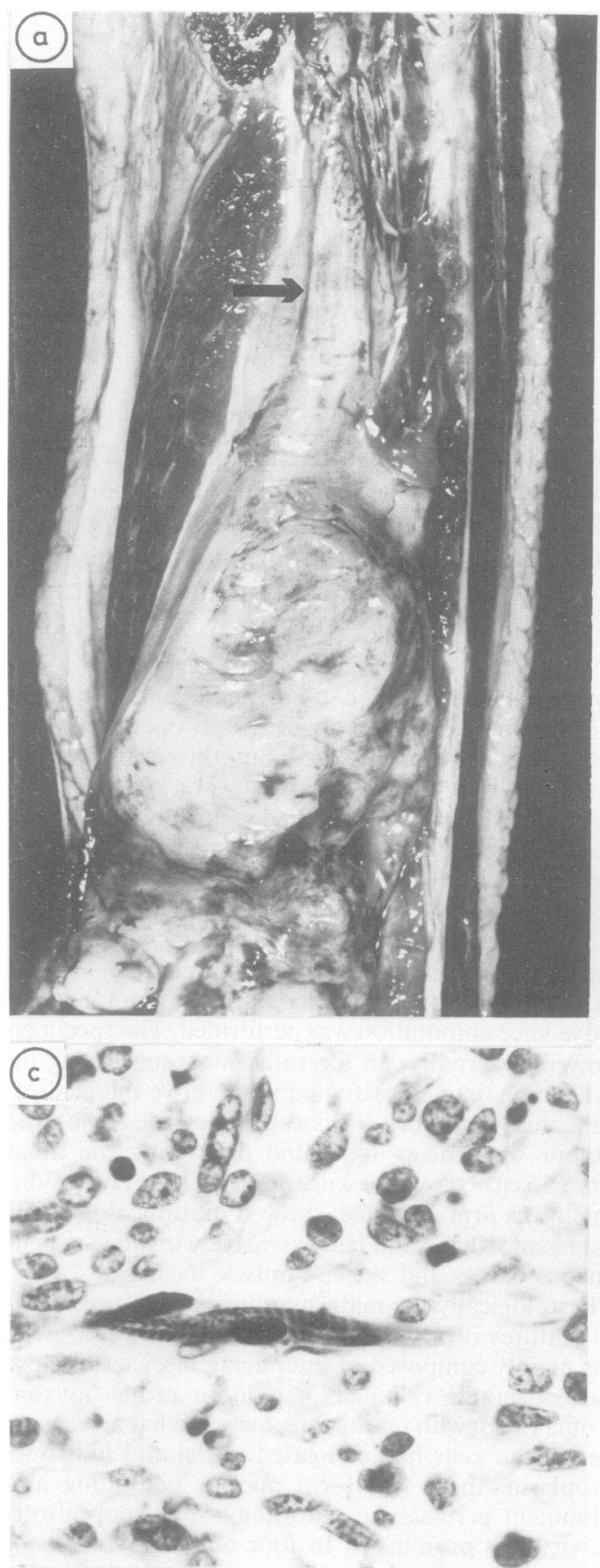
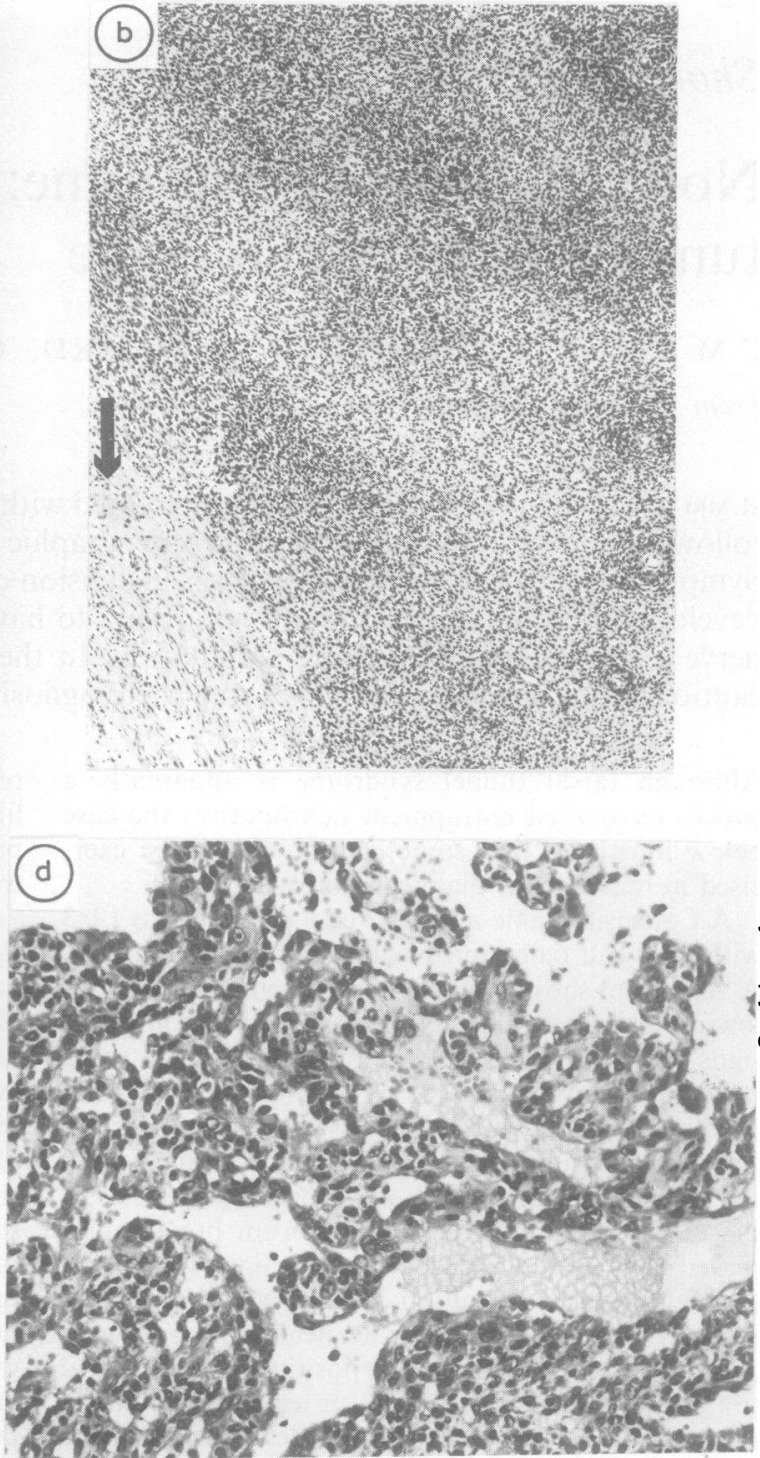

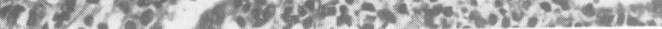

Fig (a) Arising from the tibial nerve (arrow) is a large, multinodular neoptasm showing extensive haemorrhage and necrosis. (b) Arising from nerve trunk (arrow) is a highly cellular spindle cell neoplasm, showing a fascicular arrangement $(H \& E, \times 27.5)$. (c) One of the elongated cells in a rhabdomyo-sarcomatous area shows clearly defined cross striations $(P T A H, \times 520)$. (d) Angiosarcomatous pattern with marked endothelial atypia $(H \& E, \times 114)$. 
ence of focal rhabdomyoblastic differentiation. In three other blocks there were foci of angiosarcomatous differentiation, characterised by a ramifying vascular network lined by atypical, plump and mitotically active endothelial cells which in places formed intraluminal papillae (figd). Sections were examined immunohistochemically by the immunoperoxidase technique ${ }^{2}$ for the presence of S-100 protein and with the lectin Ulex europaeus I (generously donated by Mr B Bhogal, Institute of Dermatology, London). Sections were similarly stained with antibody to-myoglobin (courtesy of Professor NA Wright, Royal Postgraduate Medical School). In the conventional spindle cell areas the tumour showed multifocal cytoplasmic activity for S-100 protein confirming its neuroectodermal origin. The eosinophilic tumour cells described above stained positively for myoglobin confirming their rhabdomyosarcomatous nature and the angiosarcomatous areas showed uniform intracytoplasmic labelling with Ulex europaeus supporting the presence of endothelial differentiation.

In summary this patient presented with a deep vein thrombosis, developed features of tarsal tunnel syndrome but subsequently was found to have a high grade malignant nerve sheath tumour arising from the posterior tibial nerve: this tumour showed rhabdomyosarcomatous differentiation (the so-called malignant Triton tumour ${ }^{3}$ ) with an additional heterologous angiosarcomatous element. He had no clinical features of neurofibromatosis.

\section{Discussion}

Tarsal tunnel syndrome occasionally results from entrapment by a tight flexor retinaculum. It is also associated with valgus deformity at the ankle, tendon sheath cysts and local trauma, ${ }^{14-6}$ and has an increased incidence in rheumatoid disease. Convincing cases however are relatively uncommon in neurological practice. The present patient appeared to have a "tarsal tunnel" syndrome which may have been precipitated by swelling and increased fluid pressure, secondary to proximal venous obstruction, under an already tight flexor retinaculum. In retrospect it is clear that both the deep venous thrombosis and signs of a tibial nerve lesion were secondary to the malignant nerve sheath tumour although no clinical, radiographic or EMG evidence suggested a proximal lesion at presentation. Magnetic resonance scanning might have suggested the diagnosis of this malignant tumour at an earlier stage. Heterologous differentation occurs in about $15 \%$ of malignant nerve sheath tumours usually being osteoid, cartilage or rhabdomyosarcoma, angiosarcoma being very rare. ${ }^{78}$ Such differentiation is more often seen in patients with neurofibromatosis though there was no evidence of this in our patient currently.

We thank Mr AE Thompson for permission to report this patient under his care.

\section{References}

1 Stewart JD, Aguayo AJ. Compression and Entrapment Neuropathies. In: Dyck PJ, Thomas PK, Lambert EH, Bunge R, eds. Peripheral Neuropathy. Philadelphia: WB Saunders \& Co, 1984:1435-57.

2 Sternberger LA. The unlabelled antibody peroxidaseantiperoxidase (PAP) method. In: Immunocytochemistry. Chichester, John Wiley, 1979:297-321.

3 Brooks JSJ, Freeman M, Enterline HT. Malignant Triton tumours. Natural history and immunohistochemistry of nine new cases with literature review. Cancer 1985;55:2543-9.

4 Edwards WG, Lincoln CR, Barrett FH, Goldner JL. The tarsal tunnel syndrome: diagnosis and treatment. JAMA 1969;207:716-20.

5 Lloyd K, Agerwal A. Tarsal tunnel syndrome, a presenting feature of rheumatoid arthritis. $\mathrm{Br} \mathrm{Med} \mathrm{J}$ 1970;3:32.

6 Goodgold J, Kopell HP, Spielholz NI. The tarsal tunnel syndrome. N Engl J Med 1965;273:742-5.

7 Enzinger FM, Weiss SW. Malignant tumours of peripheral nerves. In: Soft Tissue Tumours. St Louis: CV Mosby Co., 1983.

8 Ducatman BS, Scheithauer BW. Malignant peripheral nerve sheath tumours with divergent differentiation. Cancer 1984;54:1049-57. 